\title{
IoT based activity recognition among smart home residents
}

\begin{abstract}
Activity recognition in smart home environment is actively pursued for accessing changes in physical and behavioral profiles of home dwellers. Various activity recognition solutions have been previously proposed to implement a system with wearable sensors and smartphones. Although such solutions are widely integrated, the availability of the activity data in seamless way still poses interesting research challenges. Internet of Things (IoT) is seen as new paradigm, revolutionizing consumer electronics by extending Internet connectivity to many physical objects associated with consumer's daily life. In this paper, an Internet of Things (IoT) based activity recognition framework is proposed for activity monitoring within consumer home network. Our proposed Elgar framework handles management of activity recognition via IoT services in an IoT environment with multiple devices. The performance evaluation done pointed that the proposed system can robustly identify the activities using IoT in smart home environment with high accuracy. Hence, this system could be reliably deployed into a consumer product for the usage of home dwellers.
\end{abstract}

Keyword: Internet of Things (IoT); Smart homes; Activity recognition; Elgar framework 